\title{
Let-7a suppresses Ewing sarcoma CSCs' malignant phenotype via forming a positive feedback circuit with STAT3 and lin28
}

Jiang Xu

Nanchang University Second Affiliated Hospital https://orcid.org/0000-0002-0956-9565

Zhongzu Zhang

the Yongchuan Hospital of Chongqing Medical University

Lu Huang

Jiangxi Maternal and Child Health Hospital

Rongping Zhou

The Second Affilated Hospital of Nanchang University

\section{LinXuan Feng}

the Second Affiliated Hospital of Nanchang University

Zhenhai Zhou

The Second Affiliated Hospital of Nanjing University of Chinese Medicine

Honggui Yu

The Second Affiliated Hospital of Nanchang University

Kun Peng

The Second Affiliated Hospital of Nanchang University

Chen Li

The Second Affiliated Hospital of Nanchang University

\section{Zhiming Liu}

The Second Affiliated Hospital of Nanchang University

Kai Cao ( $\square$ kaichaw@126.com )

The Second Affiliated Hospital of Nanchang University

\section{Research}

Keywords: Ewing Sarcoma, let-7a, cancer stem cells, STAT3, lin28

Posted Date: October 27th, 2020

DOI: https://doi.org/10.21203/rs.3.rs-95105/v1 
License: (c) (i) This work is licensed under a Creative Commons Attribution 4.0 International License. Read Full License

Version of Record: A version of this preprint was published at Journal of Bone Oncology on November 1st, 2021. See the published version at https://doi.org/10.1016/j.jbo.2021.100406. 


\section{Abstract}

Background $\mathbb{Z}$ Cancer stem cells (CSCs) have been documented to be closely related with tumor metastasis and recurrence, and the same important role were identified in Ewing Sarcoma (ES). In our previous study, we found that let-7a was repressed in ES. Herein, we further identified its putative effects in the CSCs of ES.

Methods $₫$ Side population (SP) cells were isolated from ES cell lines A673 and SK-ES-1 via fluorescenceactivated cell sorting (FACS) Assays and then identified. The expression of let-7a, signal Transducer and Activator of Transcription 3 (STAT3) and lin28 were detected by Real-time qPCR and western blotting. Cell colony formation assay and cell invasion assays were performed to detected the effect of let-7a on the malignant phenotype of ES SP cells. Finally, we injected the let-7a or scramble mimic into the xenograft tumors in nude mice to evaluated its effects on the growth of ES SP cells in vivo.

Results $₫$ The expression of let-7a was consistently suppressed in the separated ES SP cells, which were identified to contain the characteristics of the stem cells. The ability of colony formation or invasion of ES SP cells was suppressed in vitro when we restored the expression of let-7a. The same results were found in the tumor growth of ES SP cells' xenograft mice in vivo. Furthermore, we found STAT3 and lin28 were involved in the suppressive effects.

Conclusion $\approx$ Let-7a, STAT3 and lin28 might form a positive circuit to regulate the malignant phenotype of ES CSCS.

\section{Background}

Ewing's sarcoma is a kind of greatly malignant primary bone tumor, which is common in children and adolescents, which progresses rapidly ${ }^{[1]}$. For patients whose lesion is localized, drug chemotherapy combined with radiotherapy or surgery can remarkably improve the survival rate to $70 \%$, but for patients whose lesion is metastatic, the survival rate is only $30 \%{ }^{[2]}$. Thus, understanding the mechanism involved in tumor metastasis and recurrence may help us enhance the survival rate of individuals with metastatic.

CSCs are produced by dysregulation of proliferation, as well as differentiation of normal stem cells, with stem-like characteristics and potential for proliferation, as well as self-renewal. CSCs is the origin of tumor occurrence, development and metastasis, although it's a small part of tumors ${ }^{[3]}$. Riggi et al. successfully isolated and identified CSCs in ES [4], they confirmed that miR-145 could down modulate the expression of OCT4, NANOG and SOX2 ${ }^{[5]}$. Meanwhile, when comparing the miRNA expression profiles of CSCs, mesenchymal stem cells (MSCs) and human induced pluripotent stem cells (iPSCs) in ES, they found that the precursors of miR-125b, miR-145, miR-30a, miR-7, let-7a, as well as miR-143 in ES CSCs, which are remarkably higher than the others. Overexpressing of miR-143 and miR-145 in ES CSCs inhibited the ability of proliferation and the growth of xenograft tumor in nude mice. The results indicated 
that miRNAs have an inhibitory effect on ES CSCs ${ }^{[6]}$, Therefore, it would make sense to explore the mechanism of miRNA in ES CSCs.

In our previously reported research, we found that let-7a function as a tumor repressor in ES cell lines ${ }^{[7]}$. As a let-7 miRNA family member ${ }^{[8]}$, let-7a has been documented to be involved in functional modulation of breast cancer stem cells ${ }^{[9,10]}$. However, its putative effect on the ES stem cells of ES remains unknown. In this study, we confirmed that let-7a represses the malignant phenotype of ES CSCs, and this function may be achieved by forming a positive feedback circuit with STAT3 and lin28.

\section{Materials And Methods}

\section{Cell line and culture conditions}

Human ES cell lines A673, and SK-ES-1 were acquired from the American Type Culture Collection and were grown in RPMI 1640 medium (Invitrogen Life Technologies, Carlsbad, CA, USA) added fetal bovine serum (10\%) (FBS; PAA, Linz, Austria), penicillin $(100 \mathrm{mg} / \mathrm{ml})$, as well as streptomycin $(100 \mathrm{mg} / \mathrm{ml})$ (Invitrogen, USA).Incubation of all the cells was conducted in an incubator $\left(5 \% \mathrm{CO}_{2}\right.$ at $\left.37^{\circ} \mathrm{C}\right)$.

\section{SP isolation and fluorescence-activated cell sorting Assays}

As documented by Goodell et al. isolation of SP was carried out as per the SP protocol (Isolation and functional properties of murine hematopoietic stem cells that are replicating in vivo.). The suspension of cells was done in DMEM $\left(1 \times 10^{5}\right.$ cells $\left./ \mathrm{ml}\right)$ enriched with $5 \% \mathrm{FBS}, 10 \mathrm{mM} \mathrm{HEPES}$, as well as $5 \mathrm{\mu g} / \mathrm{mL}$ Hochest 33342 (Sigma-Aldrich), with or without ATP-binding cassette transporter G (ABCG) repressor, Fumi-tremorgin C (10 um FTC, EMD chemicals) for $90 \mathrm{~min}$ at $37^{\circ} \mathrm{C}$. Then, incubation of the cells was conducted for $30 \mathrm{~min}$ in a shaking water bath, followed by placing the cells on ice to terminate dye efflux. After that, rinsing of the cell was carried out in $2 \mathrm{ml}$ cold HBSS enriched with FBS (5\%) (PAA, Pasching, Austria).

Excitation of the Hoechst dye was done using a krypton ultraviolet laser at 337-356 nm, and determination of its fluorescence accomplished by a 465/30 BP filter (Hoechst Blue) and a 675 BP optical filter (Hoechst Red) using a FACScalibur flow cytometer (BD Biosciences, Franklin Lakes, NJ, USA).

Sorting of the hoechst low side population cells was accomplished and subsequent incubation in serumfree complete medium consisting of DMEM medium (PAA, Pasching, Austria) enriched with $20 \mathrm{ng} / \mathrm{mL}$ EGF (Cell Signaling Tech, Denver, MA, USA), 5 rg/mL insulin, as well as $20 \mathrm{ng} / \mathrm{mL} b F G F$ (PeproTech, London, UK).

\section{Oligonucleotide transfection}

Let-7a mimic, siRNAs (distinct for STAT3, and lin28) scrambled mimic, as well as siRNA control oligonucleotides were bought from Dharmacon (Austin, TX, USA). Transfection of all the oligonucleotides into cells ( $50 \mathrm{nM}$ ) was carried out using Dharmafect 1 (Dharmacon, Austin, TX, USA) as outlined in the 
manufacturer provided protocol. The medium of the culture was refreshed via changing $6 \mathrm{~h}$ after transfection. The cells were grown another $48 \mathrm{~h}$, then harvested for evaluation.

\section{RNA extraction and RT-PCR}

For in vitro quantitation of the let-7a expression, the TRIzol reagent (Life Technologies, Darmstadt, Germany) was employed in isolating total RNA (tRNA) as per the manufacturer provided protocol. After that, gel electrophoresis was done to verify the integrity of the RNA. Then, cDNA was generated via reverse transcription of the tRNA with the First-Strand cDNA Synthesis kit (Life Technologies, Darmstadt, Germany) using gene-specific primers coupled to a TaqMan probe. Small nuclear U6 served as the internal standard. The Quanti-Tect SYBR Green PCR mixture was employed in carrying out quantitative PC, which was run on the ABI PRISM 7900 Sequence Detection System (Applied Biosystems, USA). The sequences of the specific primers for reverse transcription, as well as the RT-PCR reaction are indicated in the Supplementary Table S1. The resulting data were standardized using the internal standard, and evaluation of the relative expression levels done via the $2^{-\Delta \Delta C t}$ approach. All experiments were replicated thrice.

\section{Cell colony formation assay}

Following $48 \mathrm{~h}$ of infection, the cells were collected, followed by planting of 200 cells in a 6-well plate and allowed to grow for 14 days. The medium was changed after one week, then the medium was replaced twice a week. At the end of 14 days, rinsing of the cells was done twice in phosphate-buffered saline (PBS). Thereafter, fixing of the cells for 20 minutes was done using methanol, then 20 minutes staining in $0.1 \%$ crystal violet conducted. Lastly, cells were counted and photographed.

\section{Cell invasion assays}

All the tests were conducted in modified Boyden chambers (BD Biosciences, San Jose, CA, USA) with 8$\mu \mathrm{m}$ pore filter inserts in 24-well plates. We coated the Transwell chambers with Matrigel (BD Biosciences, San Jose, CA, USA) and solidification done for 3 hours at $37^{\circ} \mathrm{C}$. 24 hours after transfection, introduction of the $2 \times 10^{5}$ ES CSCs inserted with let-7a or scramble mimic via transfection and suspended in serumfree medium was done in the upper chamber. Furthermore, the medium enriched with $20 \%$ FBS was introduced to the lower chamber to act as a chemoattractant. At the end of $24 \mathrm{~h}$, cells retained in the upper chambers were gently removed with a cotton swab. Staining of the cells in the lower chambers was done using crystal violet, then air dried, and images acquired. All experiments were independently replicated thrice. Imaging of 6 fields from each chamber was done at $100 \times$ using on an Olympus IX81 microscope with CCD camera.

\section{Plasmid construction}

Amplification of the complete open reading frame (ORF) of STAT3, as well as lin28 was conducted, followed by cloning into the pcDNA3.1 vector to create constructs of pcDNA3.1-STAT3, as well as pcDNA3.1-lin28, respectively. Sequencing of the complete STAT3, as well as lin28 genes was done at Beijing Tianyi Huiyuan Bioscience \& Technology Inc., Beijing, China for verification of the genes. An empty 
construct of pcDNA3.1 served as a negative control. Regarding the rescue tests, ES CSCs were first inserted with constructs of pcDNA3.1-STAT3 or pcDNA3.1 $(2.0 \mu \mathrm{g})$ via transfection in 6-well culture dishes. 48 hours following transfection, we harvested the cells at intervals and assayed.

\section{Immunoblot analysis}

After $48 \mathrm{~h}$ of transfection, we harvested the ES CSCs in ice-cold PBS. Thereafter, lysing of the cells was done on ice using cold-modified radioimmunoprecipitation buffer enriched with protease repressors. The BCA Protein Assay Kit (Bio-Rad, Italy) was employed to determined the protein concentrations. After that, fractionation of the proteins was carried out on SDS-PAGE gel, then transfer-embedded onto nitrocellulose membranes (Millipore, Billerica, MA, USA). Blocking of the membranes was done for $2 \mathrm{~h}$ using non-fat milk powder ( $5 \%$ ) enriched with $0.1 \%$ Tween-20, followed by overnight-incubation with primary antibodies incubated at $4^{\circ} \mathrm{C}$ and then conjugation with the peroxidase-labeled secondary antibodies performed via another incubation. Visualization of the protein bands was done on an enhanced chemiluminescence system (Millipore, Billerica, MA, USA). The primary antibodies used herein included anti-STAT3, anti-c-Myc, anti-p-STAT3, anti-MMP2, as well as anti-lin28 (all from Cell Signaling, Danvers, MA, USA). GAPDH served as the internal standard (Zhong-Shan JinQiao, Beijing, China). All the experiments were replicated thrice.

Xenograft model assays in vivo

In the xenograft assays, subcutaneous administration of $1 \times 10^{5}$ ES CSCs was done into the posterior flanks of 12- six-week-old female nude mice. When tumors attained a size of $200 \mathrm{~mm}^{3}$, we selected 8 mice with almost equivalent volumes of tumors for subsequent experiments. These mice were randomly grouped into two groups $(n=4)$. A suspension of Let-7a or scramble mimic $(5 \mu \mathrm{g})$ in $100 \mu \mathrm{l}$ of Dharmafect 1 solution was administered into each tumor, with the injections done every 3 days for up to 7 times. After 7 days of ES CSCs injection, measurement of the tumor diameter was done, and then after every successive 3 days. After 28 days following the last administration, we sacrificed all the mice, excised the tumors, and then weighed them. At the same time, the following formula was employed to determine the tumor volume; length $\times$ width $^{2} \times 1 / 2$. The mice xenograft experiments were conducted as per the institute guidelines.

\section{Statistical analyses}

Data are indicated as mean and standard deviation. The SPSS 15.0 software was employed in computing the statistical analyses. Comparisons between 2 groups was done using the student's t-test, and analysis of variance was employed for comparison among 3 groups. Moreover, the chi-squared test was utilized for occurrence analysis. $P \leq 0.05$ signified statistical significance.

\section{Result}

\subsection{Side population sorting of ES cells and Identification}


Since SP cells were recognized as cancer stem-like cell populations, we focused to utilize this sub clone to inspect the putative influences of let-7a on the malignant phenotype of ES CSC cells. Firstly, we extracted the SP cells via staining the grown A673, as well as SK-ES-1 cells with the fluorescent DNAbinding dye Hoechst33342 and then FACS sorting. Consequently, the flow cytometric assay of Hoechstlabeled A673 and SK-ES-1 cells for blue, as well as red fluorescence disclosed a small population of cells with low fluorescence, suggesting active extrusion of the dye (Fig. 1A and 1B). The side population features of these cells with low fluorescence were validated via "normal fluorescence" (Fig. 1A and 1B) after administration with inhibitors of $A B C$ transporters, verapamil. Utilizing verapamil for characterization, $1.3 \% \pm 0.2 \%$ of the total cells in A673 culture, and $1.5 \% \pm 0.2 \%$ of the overall cells in SkES-1 growth demonstrated side population characteristics, respectively. Furthermore, to identify whether the sorted cells were malignant stem-like cells, the expression of CD133 and Sox2 was inspected. Obviously, the expression of CD133 and Sox 2 were both increased in SP cell population of A673, as well as SK-ES-1 cells (Fig. 1C), suggesting the stem-like characteristics of SP cells.

\subsection{Let-7a was repressed in SP cells of ES and overexpression of let-7a suppressed the malignant phenotype}

Our previous research has uncovered the let-7a repressive effects in ES cells. Although the repressive influences of let-7a in the CSCs of other kinds of cancers have been widely reported, its role in the CSCs of ES has not been identified. We inspected the expression of let-7a in extracted SP population of both cell lines via Taqman Realtime-PCR assays. Downstream evaluations showed that the expression of let-7a was remarkably diminished in the SP cells compared with the non-SP cells in both cell lines (Fig. 2A). Then we restored the let-7a expression in ES cells through transfected the let-7a or scramble mimic into A673, as well as SK-ES-1 cells, respectively (Fig. 2B). Then, its effects on the ratio of SP cells were performed. Interestingly, overexpression of let-7a could reduce the percentage of SP cells in ES cell lines (Fig. 2C), suggesting the putative suppressive effects of let-7a in cancer stem cell modulation in ES cells.

Then, we further inspected the influence of let-7a on the ES SP cells malignant phenotypes. The sorted SP cells were separated and cultured respectively, named as A673-SP cells, as well as SK-ES-1-SP cells. We transfected the A673-SP, as well as SK-ES-1-SP cells with let-7a mimic and scramble mimic. Evidently the expression of let-7a was markedly up-modulated in SP cells (Fig. 2D). The effects on the ability of colony formation of SP cells after transfection were tested using colony formation assays. As shown in Fig. 2E, comparing with the control group, A673-SP, as well as SK-ES-1-SP cells, overexpression of let-7a resulted in a decrease of the colony forming number. Further Matrigel invasion assays also found the same result (Fig. 2F). The group with let-7a overexpression showed less number of cells traversing through the membrane, implying that overexpression of let-7a could suppress the invasive ability of SP cells of ES. These experiments indicated that let-7a could suppress the malignant phenotype of cancer stem cells of Ewing sarcoma.

\subsection{STAT3 pathway might participate in the let-7a- mediated effects}


Signal transfer and transcriptional activator 3 (STAT3) pathway is a crucial signal pathway, which has been reported to be activated in many cancers, especially in the CSC formation ${ }^{[11,12]}$. Our previous work found that STAT3 is a direct target of let-7a and involved in let-7a-mediated repressive influences on malignant phenotype of ES cells ${ }^{[13]}$. Then, we further inspected whether it is involved in CSC formation of ES. To begin with, we explored the expression of STAT3 and its subsequent genes in A673 and SK-ES-1SP cells (Fig. 3A). Like other kinds of CSC cells, the STAT3 signaling pathway was activated in SP cell group comparing with the non-SP cells. Then, we monitored the ability of colony formation and invasion while silencing the STAT3 expression in SP cells (Fig. 3B).

The results of colony formation assays showed that cells treated with si-STAT3 remarkably repressed cell colony generation of SP cells relative to the control group (Fig. 3C). As well as transfection with let-7a, suppressing the expression of STAT3 reduced the cell number passing through the membrane (Fig. 3D), which means silencing the expression of STAT3 imitated the influences of let-7a on SP cells of ES. Since let-7a could directly target the 3'-UTR of STAT3, we suspected STAT3 might also participate in the SP cell influences. Lastly, we explored the expression of STAT3 and its downstream genes after transfection with let-7a. STAT3 expression and its subsequent genes p-STAT3, MMP2, C-myc were consistently down modulated upon transfection with let-7a (Fig. 3E). Collectively, these data further suggested the STAT3 pathway might participate in the repressive effects of let-7a.

\subsection{Upregulation STAT3 mediates let-7a repression and lin28 might participate in the modulation}

STAT3 classically behaves as an activator protein. Interestingly, in our previous paper, we found the negative relationship between let-7a and STAT3 expression, especially, we found the STAT3 expression repressed the expression of let-7a in ES cells. Thus, we also detected the expression of let-7a after suppressing the expression of STAT3. Obviously, the expression of let-7a in ES SP cells was suppressed upon transfection with STAT3 plasmid (Fig. 4A). While, it was up modulated when the expression of STAT3 was repressed (Fig. 4B). Then, there was an interesting question raised: what mediates let-7a suppression by STAT3? Except for NF-KB as previously reported, whether there were other genes involved. Lin28 is an important transcription factor that also serves a pivotal role in induced pluripotent stem cells (iPSCs) ${ }^{[14]}$. It not only performed as the important transcription factor through blocking the cracking of pri-let-7a and pre-let-7a thus inhibit the production of mature let-7a ${ }^{[15]}$, but also contain two binding sites of STAT3 on its $5^{\prime}$-UTR ${ }^{[16]}$. Overexpression of STAT3 remarkably up modulated the expression of lin28 in ES SP cells, and the contrary results were found when the expression of STAT3 was suppressed (Fig. 4C and 4D). These results suggested a putative feedback loop in ES SP cells, which is let-7a targets the STAT3 expression, and STAT3 in turn suppresses the expression of let-7a through lin28.

To identify the putative mechanism, we further explored the effects of lin28 on the expression let-7a and STAT3 in SP cells. Lin28 could block the crack of pre-let-7a and pri-let-7a and thus suppress the expression of mature let-7a. We established that overexpression of lin28 actually repressed the expression of let-7a in SP cells; conversely, knockdown its expression up modulated the expression of let- 
7a (Fig. 4E and 4F). On the other side, the expression of STAT3 up modulated correspondingly when transfected with lin28 plasmid, and the expression was suppressed upon transfection with si-lin28 (Fig. 4G and 4H). These data further suggest that the feedback loop containing let-7a, STAT3 and lin28 might perform important effects in ES CSCs.

\subsection{Restoration of let-7a represses ES tumor growth in vivo}

In order to verify the role of the positive feedback circuit in vivo, $1 \times 10^{5}$ ES CSCs were implanted into the posterior flanks of immunocompromised nude mice, with the let-7a or scramble mimic was directly administered into the resultant tumors after its formation (Fig. 5A). We observed that the volume of subcutaneous tumor injected with let-7a mimic was distinctly smaller than those injected with scramble mimic (Fig. 5B), and congruent with the tumor volume (Fig. 5C), the average tumor weight was also remarkably diminished (Fig. 5D). Immunohistochemical analysis of STAT3 protein contents in tumor tissue indicated that let-7a suppressed the expression of STAT3 in the xenografts (Fig. 5F). Finally, we also inspcted the expressions of let-7a, STAT3, and lin28 in xenografts. Congruent with our results in vitro, STAT3 and lin28 expression decreased (Fig. 5F) when let-7a expression increased (Fig. 5E) in tumors. Therefore, these data provide a theory to modulates the function of ES CSCs in ES patients through the let-7a/STAT3/lin28 circuit, which is expected to serve a positive function in the prognosis of patients with ES.

\section{Discussion}

CSCs have been documented to serve a crucial role in tumor recurrence and metastasis ${ }^{[17]}$. As to ES, the problem about recurrence and metastasis restrict the prognosis of patients for a long time ${ }^{[2]}$. Therefore, CSCs research has increasingly become an important entry point to improve the efficacy of patients with ES. Riggi et al. successfully isolated and identified CSCs in ES cells previously ${ }^{[4]}$ and confirmed the modulatory role of miRNA in ES CSCs. Meanwhile, when comparing the expression profiles of miRNA in ES CSCs and non-CSCs, they found that the expression levels of multiple microRNA precursors, including let-7a, were remarkably increased. ${ }^{[5,6]}$

As a let-7 miRNA family member, let-7a is composed of 10 distinct members (let-7f-1, 7a-1, -7a-3, -7e, $-7 \mathrm{~g},-7 \mathrm{~b},-7 \mathrm{f}-2,-7 \mathrm{a}-2,-7 \mathrm{i}$, as well as mir-98 ${ }^{[8]}$. In previous researches, we have confirmed that let-7a is under-expressed in ES tissues relative to the normal vicinal tissues ${ }^{[13]}$, and it functions in ES as a tumor repressor gene ${ }^{[7]}$. In recent years, mounting studies have shown that let-7a has an suppressive effect on $\operatorname{CSCs}^{[10,18-21]}$. Therefore, we speculate that let-7a may also play the same role in ES CSCs, thus affecting its malignant phenotype.

Tumors are composed of variety of heterogeneous cells, and CSCs as an important part of them. There were some scholars came up with an idea that CSCs are the only cells who can initiate and promote tumor growth even though they only account for a less proportion of tumors ${ }^{[22]}$. Currently, the isolation of CSCs mainly rely on cell surface specific antigen. However, as a tumor come from mesenchymal tissue, 
there is no recognized specific surface marker to ES. Therefore, we isolated ES CSCs by a method named side population cell sorting, and found that let-7a was under-expressed in ES CSCs. At present, the function of let-7a in ES CSCs and its detailed mechanism are still unclear. In our research, we established that overexpression of let-7a in ES cells lines can led to the decrease of the proportion of CSCs. In addition, overexpression of let-7a in ES CSCs, both the potential to form tumors in nude mice in vivo and the capacity of colony formation and invasion in vitro are all suppressed. These data implied that let-7a can suppress the malignant phenotype of ES CSCs.

To further explore the detailed mechanism of the let-7a in ES CSCs, we found the predict target genes to let-7a through two different kinds of miRNA target genes biology information website (miRanda and PicTar), the results showed that we got 15 target genes, the only one which have been reported associated with CSCs is STAT3 ${ }^{[11,12]}$. STAT3 is an important transcriptional activator in the JAK/STAT signaling pathway, which is related to multiple biological behaviors, e.g., cell proliferation and differentiation, gene expression, inflammatory response, and immune escape, and is also a important pathway connecting the signal transduction of cells inside and outside ${ }^{[23]}$. Some research reported that STAT3 serves a vital role in the proliferation and differentiation of CSCs of neuroglioma and breast cancer $^{[24-26]}$. In the research we had published ${ }^{[13]}$, we had confirmed that STAT3 is the target gene of let$7 a$ in ES, and in ES CSCs, we found that excessive expression of let-7a could also have repressed the expression of STAT3 pathway. These findings provide a potential mechanism to explain the suppressive effect of let-7a on ES CSCs. In order to verify our hypothesis, we silenced the expression of STAT3 in ES CSCs to detect its colony formation ability and invasion ability. The results showed that STAT3 activation had a certain influence on the ES CSC malignant phenotype. In addition, we also found that with the expression of STAT3 changed, not only the expression of let-7a, but also lin28 would make a difference.

As the one of the 4 transcription factors, lin28 is employed to iPSCs currently ${ }^{[14]}$. There are two binding sites of STAT3 on its upstream of 5'-UTR, and the results of immunoprecipitation show that STAT3 can bind to the above two sites simultaneously to up modulate the expression of lin2 ${ }^{[16]}$. Meanwhile, lin28 can bind to the let-7a precursors like pre-let-7a or pri-let-7a, blocking its cleavage and then suppresses the production of mature let-7a ${ }^{[15]}$. In addition, several researches had reported that STAT3 and lin28 are consistently highly expressed in various tumor pathological tissues ${ }^{[27,28]}$. Therefore, we put forward an assumption that maybe the lower expression of mature let-7a launched the high expression of STAT3 and lin28 in ES CSCs. Moreover, after over-expression of lin28, it will bind to the precursors of let-7a like pri-let$7 a$ or pre-let-7a, suppress its cleavage and then reduce the production of the mature let-7a. However, the low expression of mature let-7a would leads to the overexpression of STAT3 and lin28, forming a positive feedback circuit, leading to the continuous activation of let-7a/STAT3/lin28 circuit, which makes the abnormal differentiation on normal stem cells and form the ES CSCs with infinite proliferation ability, and then bring about the occurrence, metastasis and recurrence on ES. To verify our assumption, we altered the expression of lin28 in ES CSCs and detected the expression of let-7a, STAT3 and lin28 respectively. As predicted, when the expression of lin28 changed, the expression of STAT3 and let-7a showed an opposite trend. 
To sum up, we find an positive feedback circuit with a modulatory effect on malignant phenotype of ES CSCs. Because of the high rate of relapse and metastasis seriously restricts the prognosis of patients with ES, meanwhile ES CSCs as an crucial role in them, our research may provide a new train of thought and scheme for the treatment of ES, have far-reaching influence on the prognosis of patients.

\section{Conclusion}

In conclusion, the deficiency of let-7a promoted the up-regulation of STAT3, a upstream of lin28, increased the expression of lin28,and then lin28 block the release of mature let-7a furtherly. A positive circuit might formed among them which serve a pivotal role in the carcinogensis of ES CSCs. These findings maybe provide assistance for patients with ES in the future, especially those with metastasis and recurrence, and new directions for their treatment.

\section{Abbreviations}

CSCs: cancer stem cells

ES: Ewing sarcoma

SP: side population

FACS: fluorescence-activated cell sorting

STAT3: signal Transducer and Activator of Transcription 3

MSCs: mesenchymal stem cells

FBS: fetal bovine serum

ABCG: ATP-binding cassette transporter $\mathrm{G}$

FTC: Fumi-tremorgin C

ORF $\$ open reading frame

MMP2 Matrix Metalloproteinase 2

iPSCs induced pluripotent stem cells

\section{Declarations}

\section{Ethics approval and consent to participate}

All animal experiments were approved by the Ethical Institutional Review Board of the Second Affiliated Hospital of Nanchang University. 


\section{Consent for publication}

Not applicable.

\section{Availability of data and materials}

All data generated or analysed during this study are included in this published article and its supplementary information files.

\section{Competing interests}

The authors declare that they have no competing interests.

\section{Funding}

The present study is supported by the National Natural Science Foundation of China (grant no. 81460405 and 81860473), and the Innovation Driven 5511 project platform and talent team program of Jiangxi Province (grant No. 2165BCB18017).

\section{Acknowledgments}

Not applicable.

\section{Contributions}

$\mathrm{KC}, \mathrm{ZZ}$ and LH conceive the idea and designed the study. JX, RZ, LF, ZZ, HY, KP, CL performed the experimental. JX LH and ZZ participated in data acquisition and analysis. JX and ZZ co-write the manuscript. All authors read and approved the final manuscript.

\section{References}

1. Barker LM, Pendergrass TW, Sanders JE, Hawkins DS. Survival after recurrence of Ewing's sarcoma family of tumors[J]. J Clin Oncol. 2005;23(19):4354-62.

2. Balamuth NJ, Womer RB. Ewing's sarcoma[J]. Lancet Oncol. 2010;11(2):184-92.

3. Reya T, Morrison SJ, Clarke MF, Weissman IL. Stem cells, cancer, and cancer stem cells[J]. Nature. 2001;414(6859):105-11.

4. Suva ML, Riggi N, Stehle JC, Baumer K, Tercier S, Joseph JM, et al. Identification of cancer stem cells in Ewing's sarcoma[J]. Cancer Res. 2009;69(5):1776-81.

5. Riggi N, Suva ML, De Vito C, Provero P, Stehle JC, Baumer K, et al. EWS-FLI-1 modulates miRNA145 and SOX2 expression to initiate mesenchymal stem cell reprogramming toward Ewing sarcoma cancer stem cells[J]. Genes Dev. 2010;24(9):916-32.

6. De Vito C, Riggi N, Cornaz S, Suva ML, Baumer K, Provero P, et al. A TARBP2-dependent miRNA expression profile underlies cancer stem cell properties and provides candidate therapeutic reagents 
in Ewing sarcoma[J]. Cancer Cell. 2012;21(6):807-21.

7. Zhang Z, Huang L, Yu Z, Chen X, Yang D, Zhan P, et al. Let-7a functions as a tumor suppressor in Ewing's sarcoma cell lines partly by targeting cyclin-dependent kinase 6[J]. DNA Cell Biol. 2014;33(3):136-47.

8. Johnson CD, Esquela-Kerscher A, Stefani G, Byrom M, Kelnar K, Ovcharenko D, et al. The let-7 microRNA represses cell proliferation pathways in human cells[J]. Cancer Res. 2007;67(16):7713-22.

9. Sun X, Jiang S, Liu J, Wang H, Zhang Y, Tang SC, et al. MiR-208a stimulates the cocktail of SOX2 and beta-catenin to inhibit the let-7 induction of self-renewal repression of breast cancer stem cells and formed miR208a/let-7 feedback loop via LIN28 and DICER1[J]. Oncotarget. 2015;6(32):32944-54.

10. Xu C, Sun X, Qin S, Wang H, Zheng Z, Xu S, et al. Let-7a regulates mammosphere formation capacity through Ras/NF-kappaB and Ras/MAPK/ERK pathway in breast cancer stem cells[J]. Cell Cycle. 2015;14(11):1686-97.

11. Fouse SD, Costello JF. Cancer Stem Cells Activate STAT3 the EZ Way[J]. Cancer Cell. 2013;23(6):711-3.

12. Wang $X$, Sun $W$, Shen $W$, Xia $M$, Chen $C$, Xiang $D$, et al. Long non-coding RNA DILC regulates liver cancer stem cells via IL-6/STAT3 axis[J]. J Hepatol. 2016;64(6):1283-94.

13. Zhang Z, Li Y, Huang L, Xiao Q, Chen X, Zhong J, et al. Let-7a suppresses macrophage infiltrations and malignant phenotype of Ewing sarcoma via STAT3/NF-kappaB positive regulatory circuit[J]. Cancer Lett. 2016;374(2):192-201.

14. Vencio EF, Nelson AM, Cavanaugh C, Ware CB, Milller DG, Garcia JC, et al. Reprogramming of prostate cancer-associated stromal cells to embryonic stem-like[J]. Prostate. 2012;72(13):1453-63.

15. Ilopoulos D, Hirsch HA, Struhl K. An epigenetic switch involving NF-kappaB, Lin28, Let-7 MicroRNA, and IL6 links inflammation to cell transformation[J]. Cell. 2009;139(4):693-706.

16. Guo L, Chen C, Shi M, Wang F, Chen X, Diao D, et al. Stat3-coordinated Lin-28-let-7-HMGA2 and miR200-ZEB1 circuits initiate and maintain oncostatin M-driven epithelial-mesenchymal transition[J]. Oncogene. 2013;32(45):5272-82.

17. Li F, Tiede B, Massague J, Kang Y. Beyond tumorigenesis: cancer stem cells in metastasis[J]. Cell Res. 2007;17(1):3-14.

18. Yang MY, Chen MT, Huang PI, Wang CY, Chang YC, Yang YP, et al. Nuclear Localization SignalEnhanced Polyurethane-Short Branch Polyethylenimine-Mediated Delivery of Let-7a Inhibited Cancer Stem-Like Properties by Targeting the 3'-UTR of HMGA2 in Anaplastic Astrocytoma[J]. Cell Transplant. 2015;24(8):1431-50.

19. Liu Y, Li H, Feng J, Cui X, Huang W, Li Y, et al. Lin28 induces epithelial-to-mesenchymal transition and stemness via downregulation of let-7a in breast cancer cells[J]. PLoS One. 2013;8(12):e83083.

20. Sun X, Qin S, Fan C, Xu C, Du N, Ren H. Let-7: a regulator of the ERalpha signaling pathway in human breast tumors and breast cancer stem cells[J]. Oncol Rep. 2013;29(5):2079-87. 
21. Sun X, Fan C, Hu LJ, Du N, Xu CW, Ren H. [Role of let-7 in maintaining characteristics of breast cancer stem cells][J]. Xi Bao Yu Fen Zi Mian Yi Xue Za Zhi. 2012;28(8):789-92.

22. O'Brien CA, Kreso A, Dick JE. Cancer stem cells in solid tumors: an overview[J]. Semin Radiat Oncol. 2009;19(2):71-7.

23. Jin S, Mutvei AP, Chivukula IV, Andersson ER, Ramskold D, Sandberg R, et al. Non-canonical Notch signaling activates IL-6/JAK/STAT signaling in breast tumor cells and is controlled by p53 and IKKalpha/IKKbeta[J]. Oncogene. 2013;32(41):4892-902.

24. Moon SH, Kim DK, Cha Y, Jeon I, Song J, Park KS. PI3K/Akt and Stat3 signaling regulated by PTEN control of the cancer stem cell population, proliferation and senescence in a glioblastoma cell line[J]. Int J Oncol. 2013;42(3):921-8.

25. Kim SY, Kang JW, Song X, Kim BK, Yoo YD, Kwon YT, et al. Role of the IL-6-JAK1-STAT3-Oct-4 pathway in the conversion of non-stem cancer cells into cancer stem-like cells[J]. Cell Signal. 2013;25(4):961-9.

26. Chung SS, GiehI N, Wu Y, Vadgama JV. STAT3 activation in HER2-overexpressing breast cancer promotes epithelial-mesenchymal transition and cancer stem cell traits[J]. Int $\mathrm{J}$ Oncol. 2014;44(2):403-11.

27. Fan Y, Mao R, Yang J. NF-kappaB and STAT3 signaling pathways collaboratively link inflammation to cancer[J]. Protein Cell. 2013;4(3):176-85.

28. Zhou J, Ng SB, Chng WJ. LIN28/LIN28B: an emerging oncogenic driver in cancer stem cells[J]. Int J Biochem Cell Biol. 2013;45(5):973-8.

\section{Supplementary Information}

Supplementary Table S1 was not provided with this version of the manuscript.

\section{Figures}


A

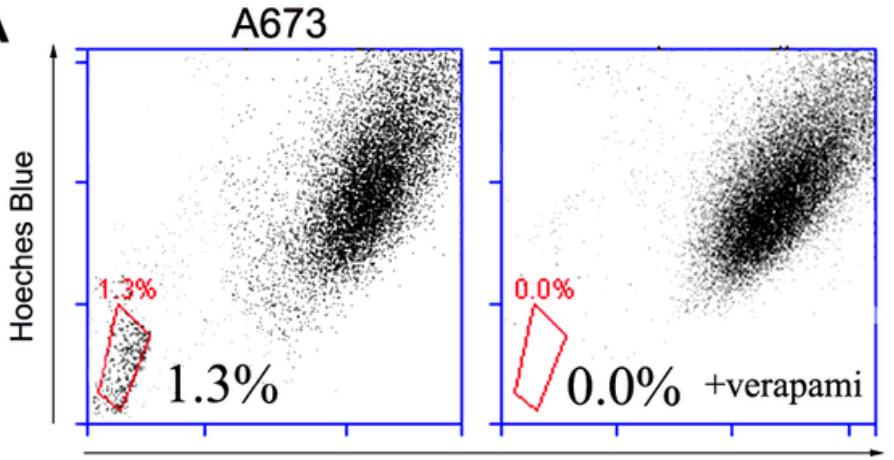

Hoechst Red
B

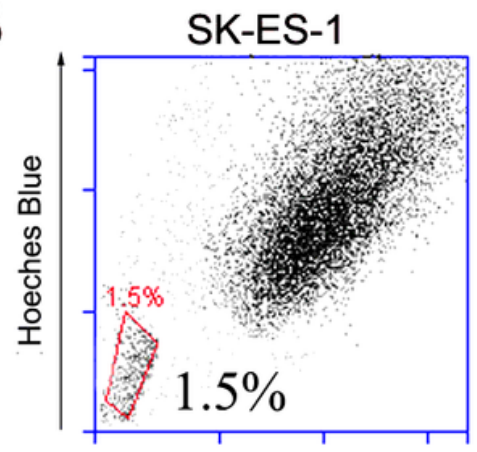

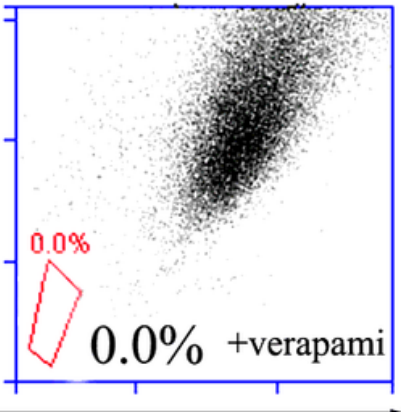

Hoechst Red

C

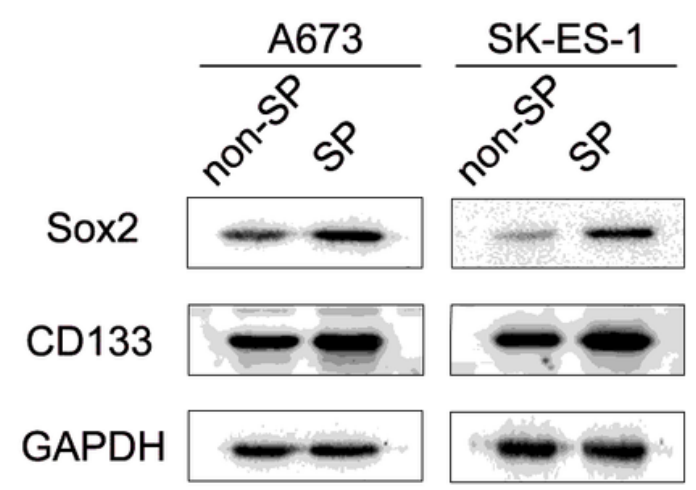

Figure 1

Side population and identification of ES cells. A and B. Flow cytometric analysis of Hoechst-labeled A673, as well as SK-ES-1 cells. Upon treated with verapamil, a part of cells with low fluorescence were sorted. C. Western blot experiments were employed to identify the expression of Sox2 and CD133 in sorted cells.

The expression of Sox2 and CD133 were increased in SP cells.

A

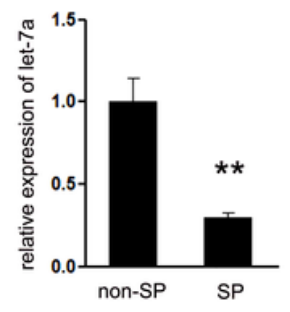

D

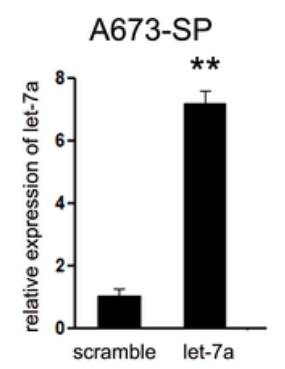

SK-ES-1
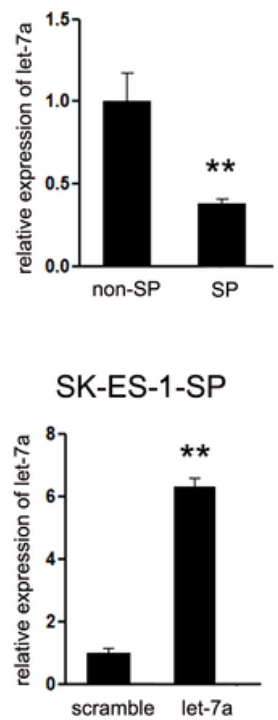

B $\quad$ A673

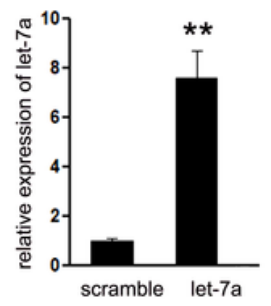

E

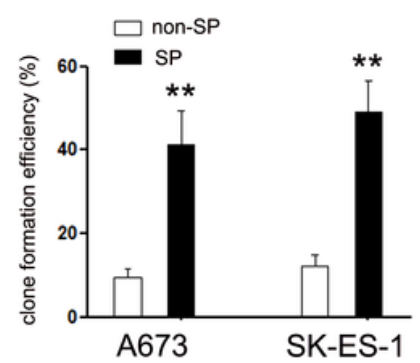

SK-ES-1

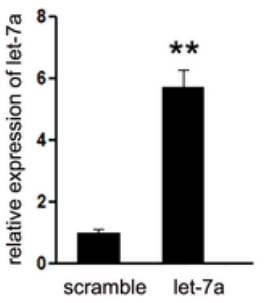

C

A673
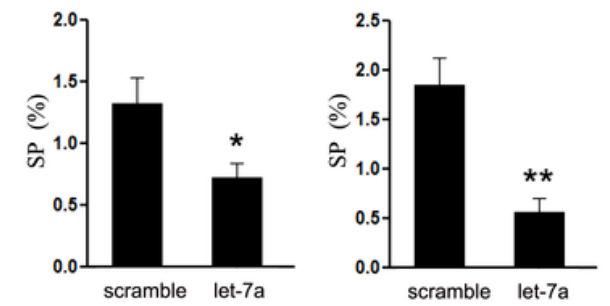

F

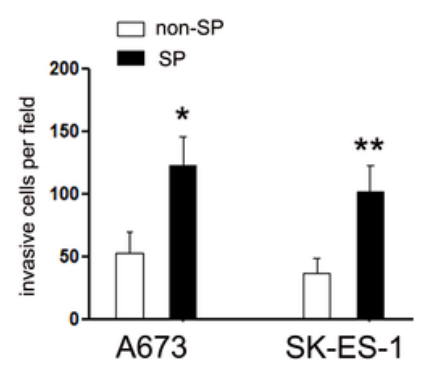

Figure 2 
Let-7a performed as a tumor repressor in the SP cells. A. RT-PCR assays were conducted to explore the expression of let-7a in the SP, as well as non-SP cells. B. The expression of let-7a was restored in sorted SP cells. C. The ratio of SP cells in ES cells upon transfection with let-7a. Upon insertion with let-7a via transfection the ratio of SP cells were decreased. D. RT-PCR assays were conducted to inspect the expression of let-7a in A673-SP cells and SK-ES-1-SP cells upon transfection with let-7a. E. The effects on the ability of colony formation of A673-SP cells and SK-ES-1-SP cells after transfection were tested using colony formation assays. Overexpression of let-7a suppressed the colony formation of ES-SP cells. F. The effects on the invasive capability of A673-SP cells and SK-ES-1-SP cells after transfection were tested. Overexpression of let-7a suppressed the cells invasion of ES-SP cells.

A

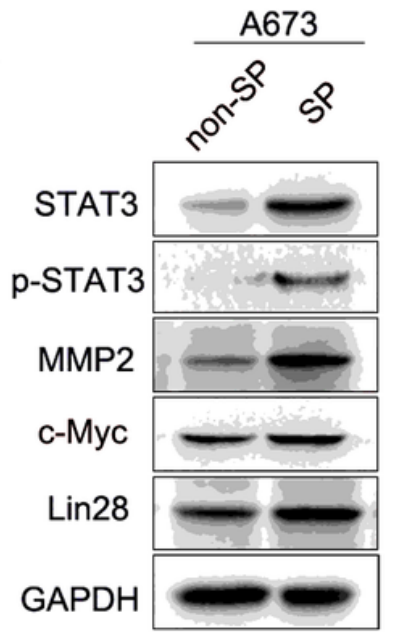

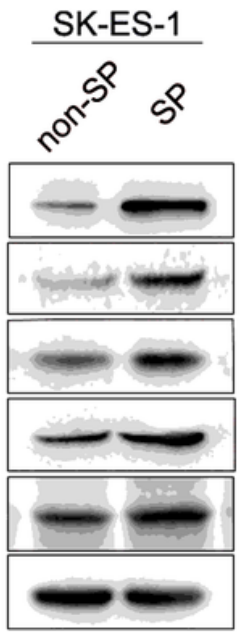

B

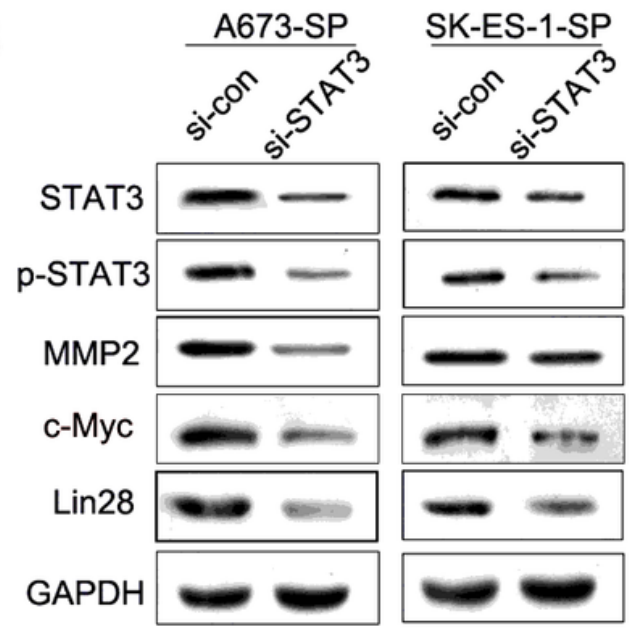

C
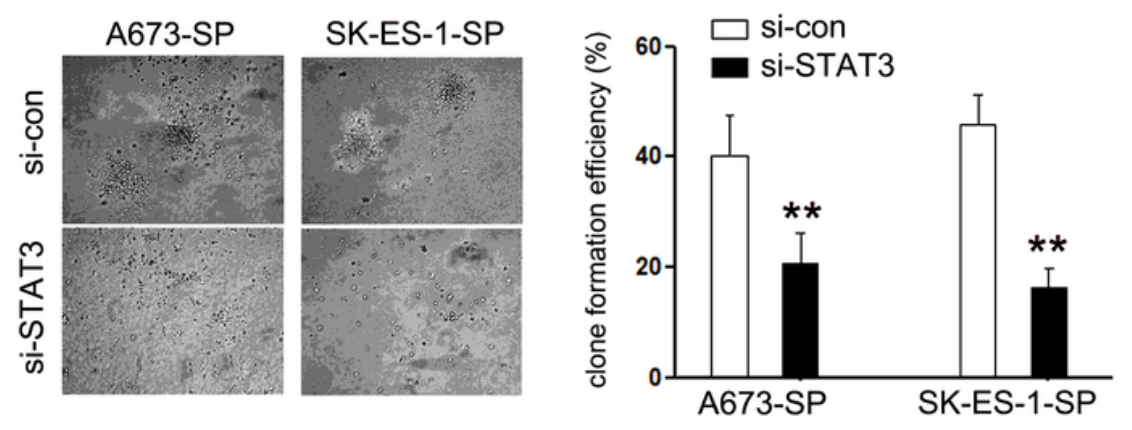

$\mathrm{D}$
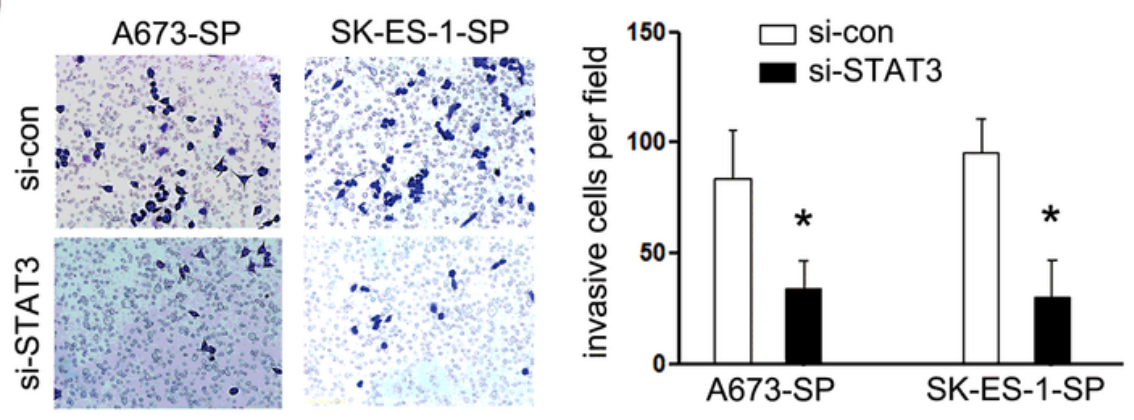

E
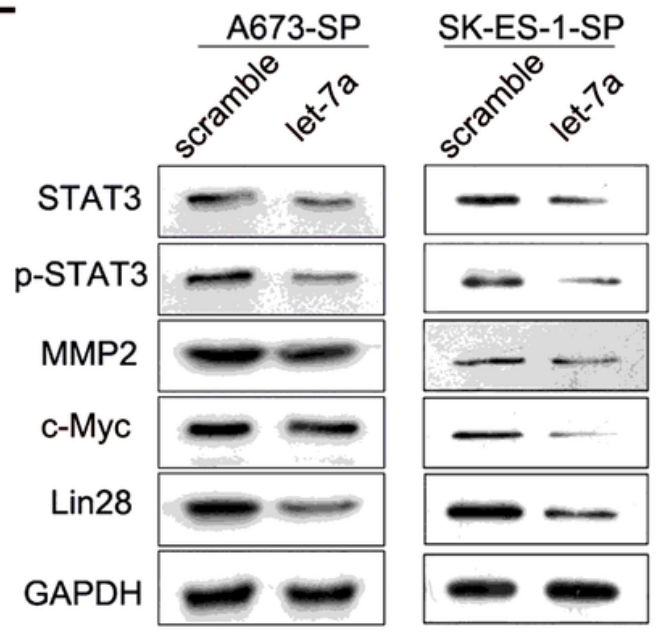

Figure 3

STAT3 might participate in let-7a-mediated repressive effects on SP cells. A. Western blot evaluation were carried out to inspect the expression of STAT3 and its down-stream genes p-STAT3, MMP2, c-Myc and lin28 in SP cells, as well as non-SP cells. B. The expression of STAT3 and its downstream genes was 
restored through transfection with si-STAT3 construct. C. The effects on the ability of colony formation of A673-SP cells and SK-ES-1-SP cells after transfection with si-STAT3 were tested using colony formation assays. Transfection with si-STAT3 suppressed the colony formation of ES-SP cells. F. The effects on the invasive capability of A673-SP cells and SK-ES-1-SP cells after transfection with si-STAT3 were tested. Transfection with si-STAT3 suppressed the cells invasion of ES-SP cells. E. The expression STAT3, $p$ STAT3, MMP2, c-Myc and lin28 were tested in SP cells upon insertion with let-7a via transfection.
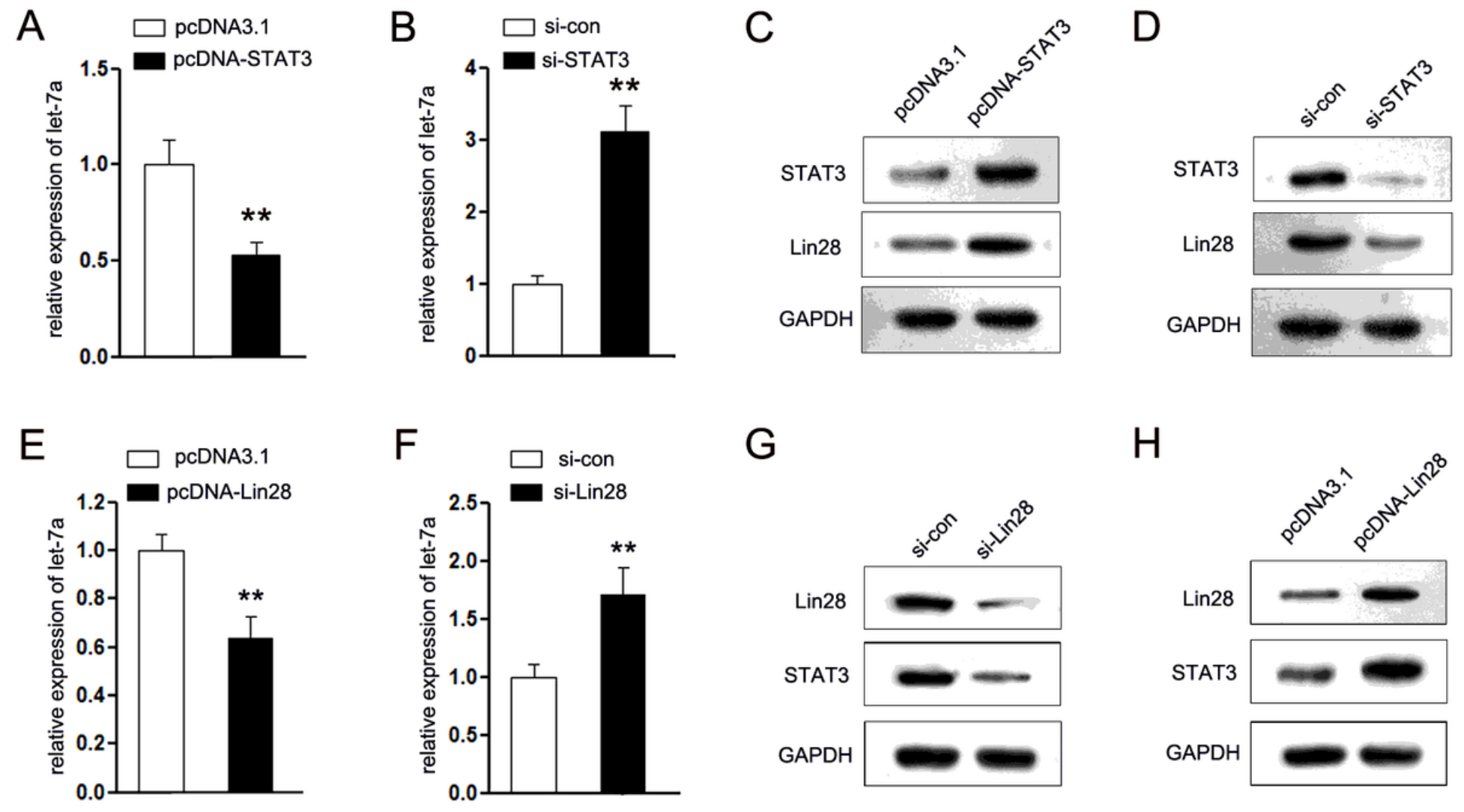

Figure 4

STAT3 could modulate the expression of let-7a through lin28 in SP cells. A. RT-PCR inspection showed the expression of let-7a was suppressed in A673-SP cells upon restored the expression of STAT3. B. The expression of let-7a was up modulated in A673-SP cells upon repressed the expression of STAT3. C. Western blot assays showed the expression of lin-28 was increased upon transfection with STAT3. D. Western blot evaluation showed the expression of lin-28 was suppressed upon transfection with siSTAT3. E. RT-PCR analysis demonstrated the expression of let-7a was suppressed in SP cells upon transfection with lin28. F. RT-PCR assays showed the expression of let-7a was up modulated in SP cells upon transfection with si-lin28. G. Western blot analysis indicated the expression of STAT3 was up modulated upon transfection with lin28. H. Western blot inspection showed the expression of STAT3 was decreased upon transfection with si-lin28. 

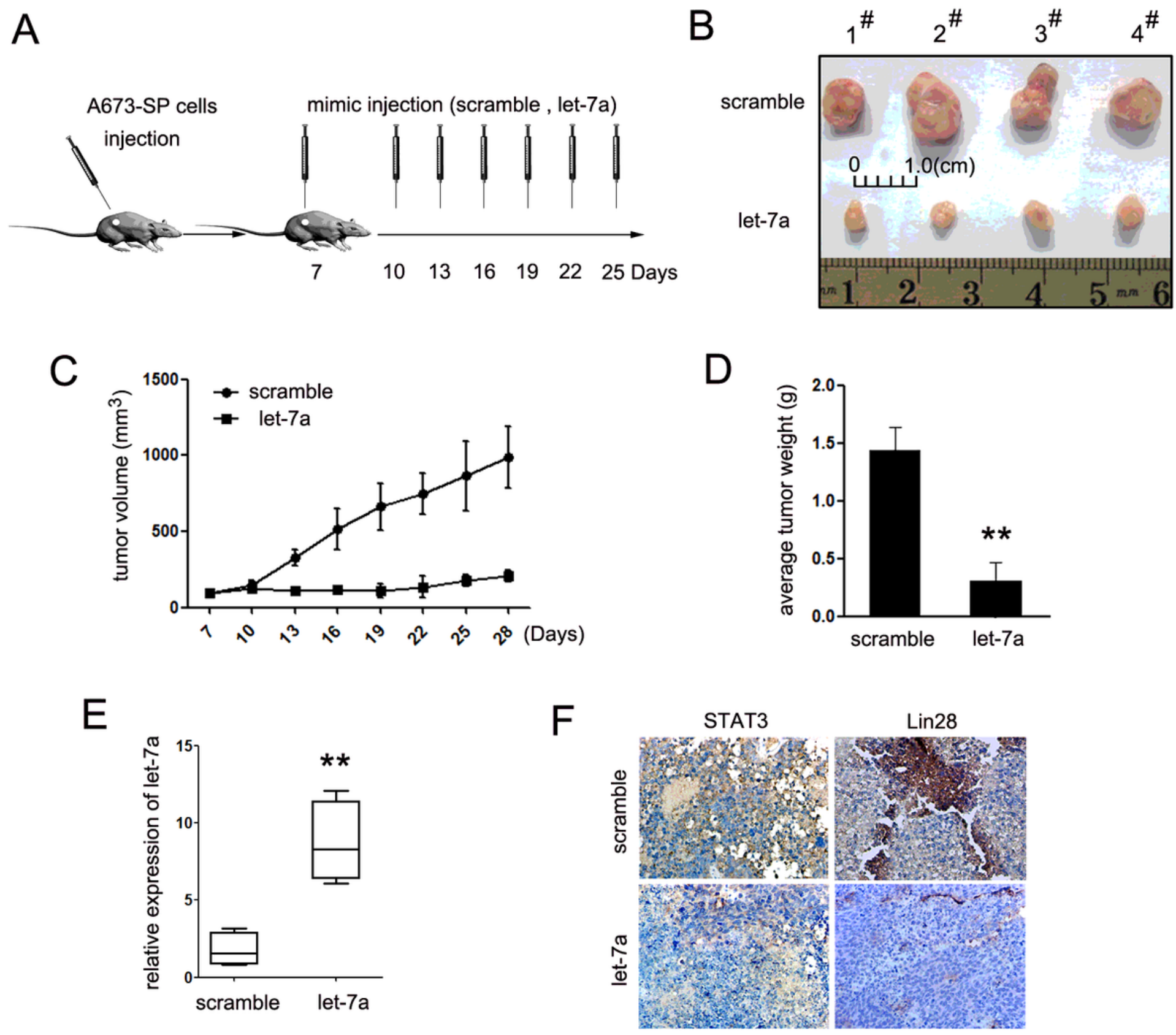

Figure 5

Let-7a suppressed the tumor growth of SP cells in vivo. A. Diagram indicating the experimental procedure employed in the mouse xenograft experiments. (B) Illustrative images of treated mice, as well as 4 xenograft tumors. ( $C$ and D) Graph illustrating the average tumor volume, as well as the weight for each mouse groups at the end of the experiment. $(E)$ Let-7a expression in xenografts from mice injected with A673 cells transfected with and without let-7a mimic. (F) Immunoblot analysis of lin28 and STAT3 in tumors from xenograft mice. 